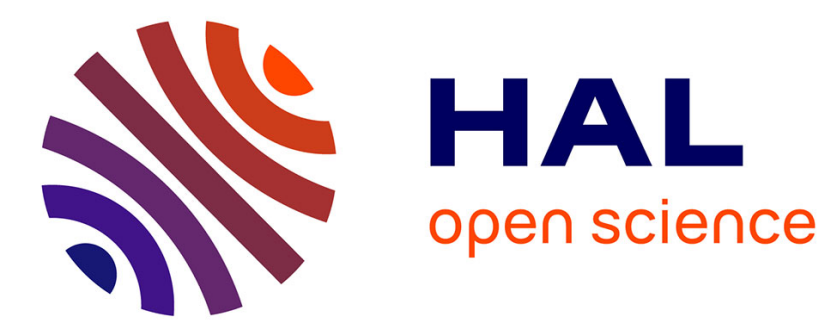

\title{
Human-humanoid interaction and cooperation: a review
}

Lorenzo Vianello, Luigi Penco, Waldez Gomes, Yang You, Salvatore Maria

Anzalone, Pauline Maurice, Vincent Thomas, Serena Ivaldi

\section{To cite this version:}

Lorenzo Vianello, Luigi Penco, Waldez Gomes, Yang You, Salvatore Maria Anzalone, et al.. Humanhumanoid interaction and cooperation: a review. Current Robotics Reports, 2021, 2 (4), pp.441-454. 10.1007/s43154-021-00068-z . hal-03413650

\section{HAL Id: hal-03413650 \\ https://hal.science/hal-03413650}

Submitted on 3 Nov 2021

HAL is a multi-disciplinary open access archive for the deposit and dissemination of scientific research documents, whether they are published or not. The documents may come from teaching and research institutions in France or abroad, or from public or private research centers.
L'archive ouverte pluridisciplinaire HAL, est destinée au dépôt et à la diffusion de documents scientifiques de niveau recherche, publiés ou non, émanant des établissements d'enseignement et de recherche français ou étrangers, des laboratoires publics ou privés. 


\title{
Human-Humanoid Interaction and Cooperation: a Review
}

\author{
Lorenzo Vianello ${ }^{1,2 \dagger}$, Luigi Penco ${ }^{1 \dagger}$, Waldez Gomes ${ }^{1}$, Yang \\ You $^{1}$, Salvatore Maria Anzalone ${ }^{3}$, Pauline Maurice ${ }^{1}$, Vincent \\ Thomas $^{1}$ and Serena Ivaldi ${ }^{*}$ \\ 1*Inria, Loria, Université de Lorraine, CNRS, Nancy, F-54000, \\ France. \\ ${ }^{2}$ CRAN, Nancy, F-54000, France. \\ ${ }^{3}$ Laboratoire CHArt, Université Paris 8 , Paris, F-93200, France.
}

*Corresponding author(s). E-mail(s): serena.ivaldi@inria.fr;

Contributing authors: lorenzo.vianello@univ-lorraine.fr; luigi.penco@inria.fr; waldez.azevedo-gomes-junior@inria.fr;

yang.you@inria.fr; sanzalone@univ-paris8.fr; pauline.maurice@loria.fr; vincent.thomas@loria.fr;

$\dagger$ These authors contributed equally to this work.

\begin{abstract}
Purpose of review: Humanoid robots are versatile platforms with the potential to assist humans in several domains, from education to healthcare, from entertainment to the factory of the future. To find their place into our daily life, where complex interactions and collaborations with humans are expected, their social and physical interaction skills need to be further improved. Recent findings: The hallmark of humanoids is their anthropomorphic shape, which facilitates the interaction but at the same time increases the expectations of the human in terms of advanced cooperation capabilities. Cooperation with humans requires an appropriate modeling and real-time estimation of the human state and intention. This information is required both at a high-level by the cooperative decision-making policy and at a low-level by the interaction controller that implements the physical interaction. Real-time constraints induce simplified models that limit the decision capabilities of the robot during cooperation.
\end{abstract}


Summary: In this article, we review the current achievements in the context of human-humanoid interaction and cooperation. We report on the cognitive and cooperation skills that the robot needs to help humans achieve their goals, and how these high-level skills translate into the robot's low-level control commands. Finally, we report on the applications of humanoid robots as humans' companions, co-workers or avatars.

Keywords: humanoid robots, human-robot interaction, cooperation

\section{Introduction}

Employing humanoid robots in real-world scenarios is still a challenge because of the inherent complexity of locomotion, balancing and interaction with humans or unknown environments. However, their versatility and their anthropomorphism makes them the ideal platform to operate in environments conceived and designed for humans. Several research projects and international competitions have highlighted the potential of humanoid bipedal technologies $[1,2]$ : with their extended mobility in unstructured or cluttered environments, they can be used in a range of different applications, such as search and rescue, disaster response [1] and aircraft manufacturing [2]. In the last decade, the advances in mechatronics technologies made it possible for companies such as Honda, Boston Dynamics and Agility Robotics to produce prototypes and commercial platforms (ASIMO [3], Atlas [4] and Digit [5], respectively) that showcase the level of maturity required for robots to operate in the real world. Yet, further advancements are required to integrate such robots into our daily lives, where complex interactions and collaborations with humans are expected.

In contrast to the structured working space of the laboratory or production/testing site, the environment in which the robots are expected to operate is characterized by a high level of dynamic uncertainty, and by the presence of several human collaborators or bystanders. In this context, the robot must be able to socially and physically interact with the human counterparts. It also needs to exhibit advanced cognitive interaction skills to cooperate with human workers, assisting them in their tasks. Figure 1 illustrates some examples of human-humanoid interaction and cooperation, where the robot's degree of copresence ranges from remote (e.g., in teleoperation) to proximal, even in the human's peripersonal space.

In this article, we review the key elements of human-humanoid interaction and cooperation. We present and analyze the current achievements from a human-centered point of view, considering that a humanoid robot interacting with humans should help them to improve their health or working conditions. Figure 2 shows the interconnection between the key building blocks that enable a robot to make complex decisions and take actions to cooperate with a human: it is meant to guide the reader through the different topics and sections. In 

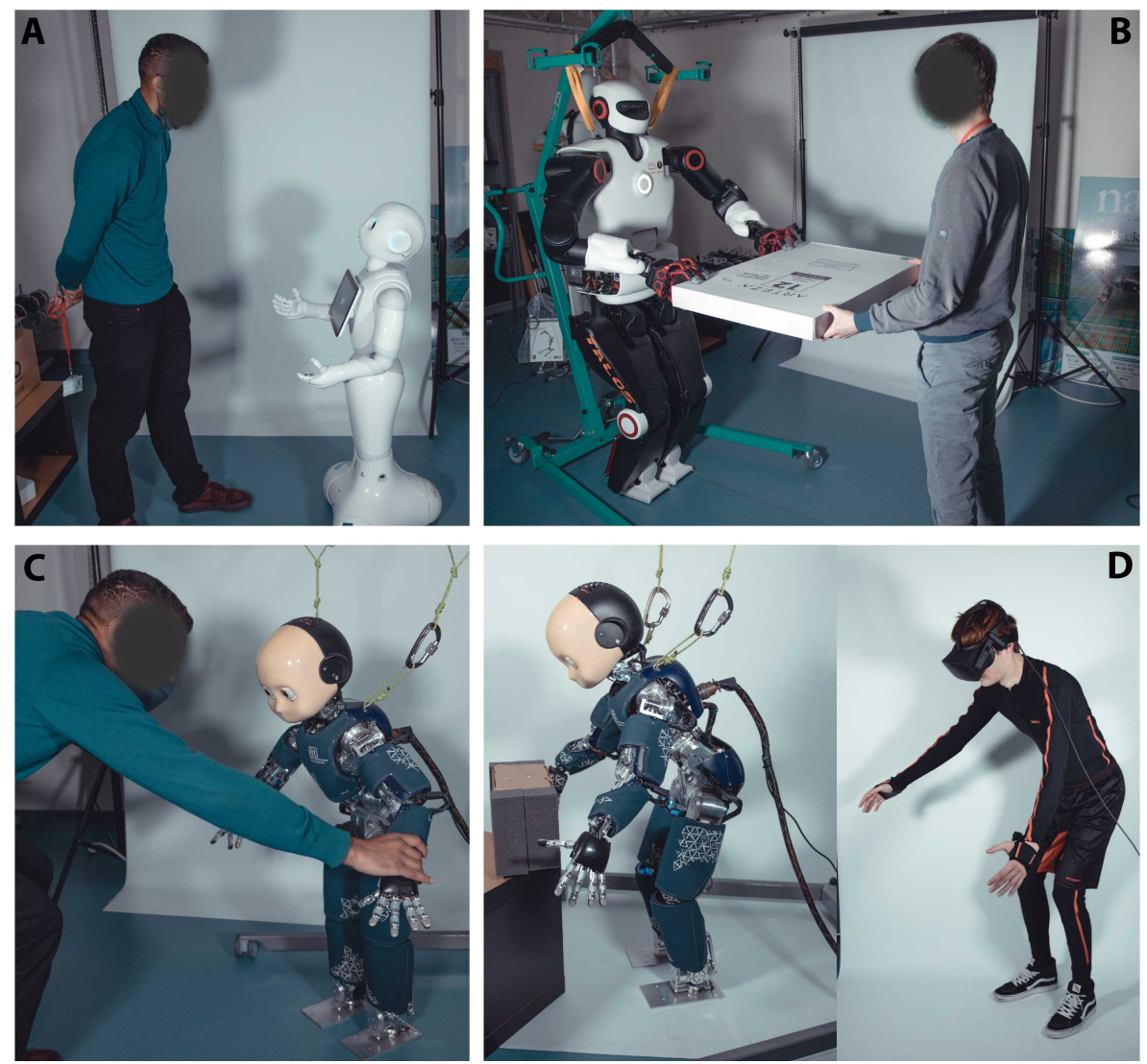

Fig. 1 Examples of human-humanoid interaction and cooperation: A: social interaction with a Pepper; B: cooperative load carrying with a Talos; C: passive physical interaction with an iCub; D: iCub teleoperated by a human operator.

Section 2 we discuss the principal issues in building cognitive and social skills. In Section 3 we formalize the concept of physical human-robot cooperation, while in Section 4 we overview the main interaction control approaches that enable low-level physical interaction between the robot and the human. The knowledge of the human "state" is required by both the high and the low level control to build human-aware control plans: hence we present the main methods used to model and perceive the humans in Section 5. Finally, in Section 6, we report on the current main application of humanoid robots interacting and cooperating with humans: humanoids as personal assistants, co-workers and avatars.

\section{Social and cognitive interaction}

Endowing humanoids with cognitive skills is a pivotal step to safely blend them in our society. Such skills go beyond the abilities of reasoning, exploration 
and learning, and are rather oriented towards a mutual interplay between the humanoid "brain", its physical embodiment and its environment. In this sense, cognitive skills emerge from a proper and coherent exploitation of stochastic internal models of the knowledge the robot has of itself and of its surrounding. These models mediate past knowledge with new perceptions and are continuously and incrementally updated according to feedback from new experiences [6]. As inherently probabilistic, the models are not only able to represent temporal information through short and long-term memories [7], but also spatial deictics [8] , surmounting the limits of the Cartesian definition of space, embracing the more flexible concepts of "here" and "there" and of "this, "these", "that", and "those".

A consistent social interaction is achieved when the robot is perceived by the human partners as "believable" through its appearance and through the consistency of its actions and its social behaviors. Any physical or behavioral inconsistency can be quickly spotted, perceived as "strange", making the robot become unacceptable for the human partners.

Because of their anthropomorphic appearance, humans tend to spontaneously attribute social intelligence to humanoid robots [9]. Designers can exploit and enhance this tendency, inducing in partners the projection of traits, emotions and intentions typically associated with humans. However, even if making robots more appealing and acceptable, anthropomorphism raises the expectation people have about their actual cognitive abilities. In this sense, the behavior consistency requested to humanoids is not limited to a coherent sequence of actions, but it is extended to the challenge of being "readable", "legible" and "predictable" by human partners [10]: the robot reveals its intentions and its internal state through a coherent production of verbal and non-verbal social cues. Achieving coherent robot behaviors in response to the human reactions strictly relies on the production of metrics, models, techniques and algorithms aimed at capturing and describing the dynamics of the social interplay [11], and on the development of robot "socio-cognitive" skills capable of explicitly taking into account the human presence in their perceptioncognition-action loop [12]. This socio-cognitive skill will endow humanoids with a sort of theory of mind [13], making them able to attribute mental states, intents, emotions and personalities to themselves and to the others. Models based on such concepts have already shown their potential and can be particularly interesting for achieving engagement in long-term interactions [14], where the customization of behaviors becomes central.

The feeling of "illusion of life" [15], elicited by the coherence between the humanoid's embodiment, its actions and its social behaviors, can vary among the scenarios in which such robots are employed, among category of users and among people, being strictly dependent on the bias they have towards humanoids, towards robots and, more in general, towards technology.

To evaluate how people perceive robots, several questionnaires have been proposed, such as Godspeed [16], and Negative Attitudes Toward Robots Scale 


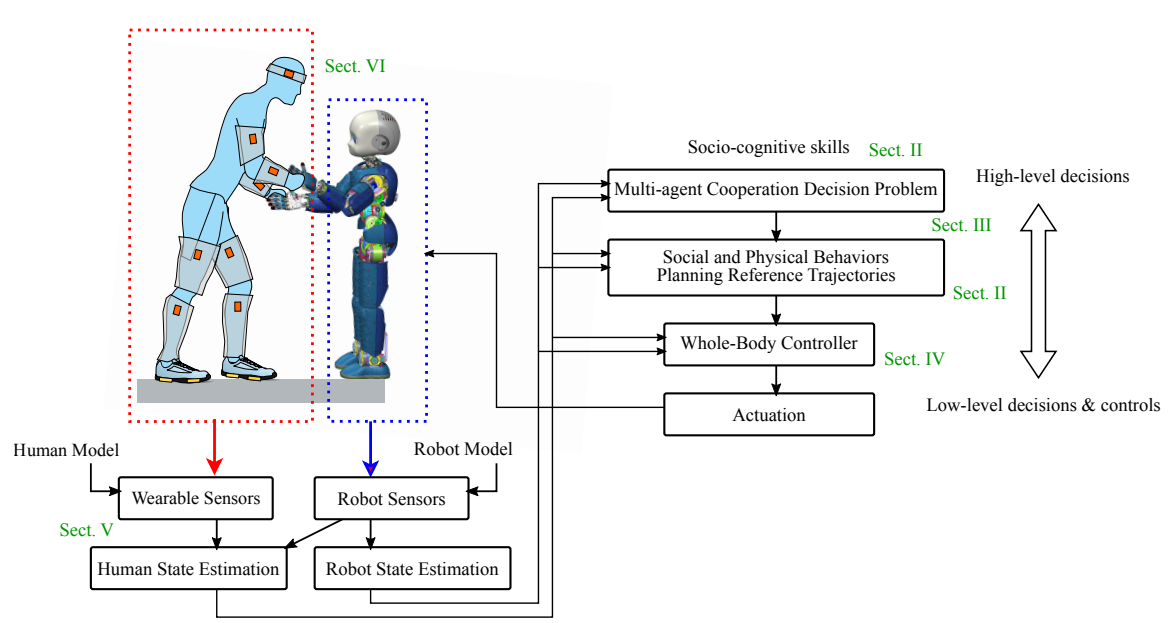

Fig. 2 Schematic drawing of the components involved in Human-Humanoid Interaction and cooperation.

(NARS) [17]. Results from such questionnaires, together with human's behavioral metrics, are extremely useful tools to evaluate the effectiveness of the social interplay with humanoids in real-world scenarios [14].

\section{Cooperation: a decision problem}

Social and cognitive skills are critical to make the robot believable and to ensure it can perform in day-to-day interactions to significant endeavors. These skills are the building blocks that enable a humanoid robot to pro-actively cooperate with humans and help them achieve their goals. Developing these skills requires a human-centered design of the robot's intelligence, that at many decision levels should integrate the goals and costs of the human's actions to plan suitable high-level assistance actions and translate those decisions into the robot low-level control commands. Figure 2 shows the interconnection between these skills: to cooperate, the robots needs to formulate the problem of finding the best sequence of actions that assist the human in achieving their goals and minimizing their costs, considering the constraints and limits of the robots and the human as individuals first, then as interacting agents. This requires also cognitive reasoning, in particular taking the human perspective [13]. Solving this problem requires high-level decision making capabilities, which are then translated at a lower level into the modules producing social and physical interaction behaviors, also translated into lower-level motor commands for the robot.

Several approaches are possible to design this robot high-level decision making system. Among those approaches, the robot's policy can be designed based on expert knowledge or directly learnt through interactions with a 
human, however, in both cases, this would require to encompass all the possible encountered situations and the variety of human reactions (needing a complete knowledge of all the possible situations in the first case or an important amount of data in the second one). In this section, we propose specifically to consider the use of a planning approach to compute the high-level robot's intelligence.

Whereas a planning approach requires a representation of the interaction situation, a detailled knowledge of its dynamics, and an adaptation of the highlevel strategy to low-level controls, it still seems a promising direction to us: (1) it can leverage generic models and algorithms to automatically compute a robot's strategy from the definition of the collaborative task, (2) planning approaches are able to deal with several sources of uncertainties like sensor noises or uncertainties regarding the human behaviour or mental state, (3) planning approaches are generic and various questions of paramount importance for collaborative humanoid robotics have been represented and can merged in that same framework like intention estimation [18], role attribution and/or inference of user profile [19], and, (4) planning allows to compute strategies considering the long-term consequences of the robot's behaviour which could have a huge impact in the decision process (e.g., when optimizing the user's fatigue).

More precisely, in a planning context, a collaboration problem can be modelled as a Multi-Agent sequential decision problem where two agents, the human and the robot, select actions according to their respective policies in a coordinated way to achieve a common task, where typically cumulative shared rewards represents the common goal. This problem can typically be addressed in game theory framework, defining the agents' strategies and the rewards.

Due to the difficulty in modelling the complex and often unpredictable human behavior, the most common formulations of human-robot interaction and cooperation resort to single-agent problems: they only consider the robot's point of view, while the human is assumed as part of the environment and modelled as purely reactive agent with a known policy [20]. Solving a singleagent problem consists in building the long-term robot's policy and potentially influencing the human reactions in order to complete the collaborative task in the most efficient way [21].

The robot decision problem is often formalized by a Partially Observable Markov Decision Process (POMDP), a general framework well suited to model different collaboration situations faced by the robot: it assumes that the robot is acting in an uncertain environment, described by a Markov Decision Process (MDP) but the robot cannot directly observe its underlying state. The uncertainty of the human reaction can be represented by considering a stochastic evolution of the system. A POMDP is usually defined by the tuple: $<S, A, T, \Omega, O, r, b_{0}>[22]$. At each time step, the agent is in a state $s \in S$ performs an action $a \in A$ influencing the evolution of the state of the system according to a probability distribution $T\left(s, a, s^{\prime}\right)=P\left(s^{\prime} \mid s, a\right)$ and receives an observation $o \in \Omega$ depending on the new state that has been reached and the 
observation function $O(o, s)=P(o \mid s)$. The agent has no direct access to the state of the system but only to those observations. Using past observations, the agent infers a belief-state, a distribution of probability over the true state of the system, and makes their decision based on this estimate.

Several algorithms can be used to build the optimal robot policy (e.g. [23]), which may also include actions to gather information about the human state and simultaneously estimate the hidden variable conditioning their actions (e.g., their profile or objective) [24]. As such, POMDPs are ideal to develop collaborative robot strategies in absence of structured rewards or structured cooperation instructions.

One of the main difficulties in using this approach lies in the modelling of the human behaviour: the critical questions are how to consider the expectation of the human towards the robot and how they will adapt their policy depending on the robot's actions. Often, the interaction between human and robot is structured along "roles", .e.g, leader/follower, which may determine not only high-level decisions but also low-level actions (e.g., stiffness in impedance control). For example, in a strictly asymmetric leader-follower case, the humans cognitive abilities can be used to supervise or to lead the robots superior physical capabilities; whereas in an egalitarian roles distribution, where the leadership is not specified, the robot may need to continuously adjust its own role, and consequently its behavior, according to the humans intention and estimated role [25].

Another difficulty is defining the right rewards, so that the robot policy can truly help the human to achieve their goal. This is not an easy task since, usually, the utility we would like to optimize is not reduced to a single dimension and must consider all possible criteria. It might involve the efficiency of the task achievement but also the human ergonomics and physiological comfort, the cognitive load, as well as unknown objectives [18]. This is a problem of paramount importance due to reward hacking problems [26]: the produced policy will optimize the given reward but might have unexpected side effects that could be counter-productive or dangerous.

Building policies able to simultaneously estimate the hidden variables determining the human reactions and consider long-term consequences of the robot actions are a key component to build adaptive robots that collaborate with humans in a proficient way. Those models will turn out to be very useful in assistive robotics in order to adapt the robot behaviour to the user profile and to their physiological status.

\section{Motion control for physical interaction}

Once the cooperation strategy with the human has been defined, and the robot has access to the human state estimation, it has to be controlled adequately to enable the physical interactions. The previous section discussed how the robot can plan for cooperative actions, at the high level, taking into account the human's goals and states. High-level decisions must be translated into 
low level commands, typically by means of desired behaviors, implemented as desired trajectories, which needs to be translated into motor commands, as represented in Figure 2. The critical aspect that distinguishes a robot motion controller for cooperation with humans from a generic one for the robot alone in the environment is to consider the human in the design of the motion control, i.e., to design a "human-aware" controller [27]. This means to consider the human state, their dynamics [28], their intended movement [29], and use the predictions of their future states to plan suitable robot motions and physical interactions. These interactions often result in complex behaviors, where the humanoid needs to simultaneously control various aspects of its internal and external motion like locomotion, posture, gaze, manipulation and contact stability. All these aspects are usually considered as different tasks and the whole-body controller becomes a multitask optimization problem. This is classically formulated as a Quadratic Program (QP), where the control input (the motor torques, the joint positions or velocities) is found so to minimize a multivariate quadratic function related to the difference between the actual value of the tasks and their references. The QP formulation provides extreme flexibility in the choice of the type of control, allowing one to solve inverse kinematics [30], inverse dynamics [31] or momentum-based [27] control problems for both position-controlled and torque-controlled humanoid robots.

In dynamic environments and in the presence of humans, momentum-based control methods on torque-controlled robot are generally the preferred choice. These approaches in fact, can ensure safety and contact stability under unexpected physical interactions [32], so they are appropriate to handle possible collisions between the robot and the humans during the robot's trajectory execution. In other situations, the robot has to adapt its motion to that of the human, considering some variability in the execution of the various task references and considering perceived external forces. In this case, to achieve a stable robot behavior while maintaining contacts with the human, compliant control approaches such as impedance control [33], and admittance control [34] have been applied. Early works proposed separation of tasks: impedancebased upper body manipulation with lower body balancing [35]. However, this solution is not well suited for bipedal walking under physical interactions and whole body control is better suited [31].

During interaction, one key-point for the robot is the computation of the reference task: it usually defines the robot motions, but it may also be used to influence the human motion through physical contact. An optimized choice of the interaction trajectory (for instance, at the end-effector) could be used to reduce the human effort [36] and make the cooperation more comfortable and safe [37]. This computation is highly dependent on the estimated human intention. Following the terminology of [36], we could divide the robot strategies in reactive and proactive. In reactive strategies the belief of human reference task is computed online and the robot react accordingly. These kind of approaches are highly dependent by the sensors integrated in the robot, for this reason a discussion about them has been presented in Section 5. On the other hand, 
in proactive strategies, the robot predicts a long-term belief of human reference task and plan solutions accordingly [38]. Once the robot has access to the human current (reactive) or long-term (proactive) intention, it can use the information to compute its own reference task. The final task computation depends on the human intention and the estimated role as cooperative agent [39].

A limitation of most existing controllers for physical human-robot interaction is the representation of the human state, which is vastly simplified as an external force or end-effector's pose; this limits the quality of the solutions proposed by the robot and the extent to which the robot can reason about the human. For this reason, recent work [27] proposed to extend the classical quadratic program whole-body formulation in the case of human-humanoid physical interaction, and to include the human model in the system model. This allows to reason about the whole-body dynamics of the human, considering their dynamics (e.g., joint torques), their posture and even ergonomics-related quantities that may be instrumental to ensure a safe and ergonomically optimized collaborative motion. One key issue is how to model the human kinematic and dynamic properties. Depending on the extent and objectives of the cooperation, humans could be represented by a simple linear inverted pendulum [40] or a more complex Digital Human Model (DHM) [27]. Choosing the correct level of abstraction and simplification in the human model can make the difference in real-time performance. In the next section, we will discuss how to model the human and how to estimate their state based on multi-modal sensor measurements.

\section{Human perception and modeling}

Similarly to other cooperative robots, humanoid robots need to estimate the human physical, physiological and cognitive state in order to collaborate with their human partner effectively: as discussed in the previous sections and shown in Figure 2, the human state is a critical input for the high-level decision planning and lower level motion planning and control. The perception of the human state relies on sensors that can be placed in the environment, embedded on the robot, or worn by the human. A list of sensors commonly used to perceive humans is presented in Table 1. State-of-the-art motion capture techniques remain widely used to provide high-fidelity and high-frequency measurements of human kinematics. While human kinematics can serve to inform about human's intent, the on-line estimation of human dynamics is receiving a lot of attention since it enables the robot to consider aspects such as balancing, or humans' internal force distribution [43]. Dynamics estimation requires a measure of external forces, either via generic force/torque $(\mathrm{F} / \mathrm{T})$ sensors that can be embedded in the robot, or via specific sensors such as force plates for human/ground reaction force. Wearable force sensors such as sensorized insoles are also of interest due to their portability [45]. 
10 Human-Humanoid Interaction and Cooperation: a Review

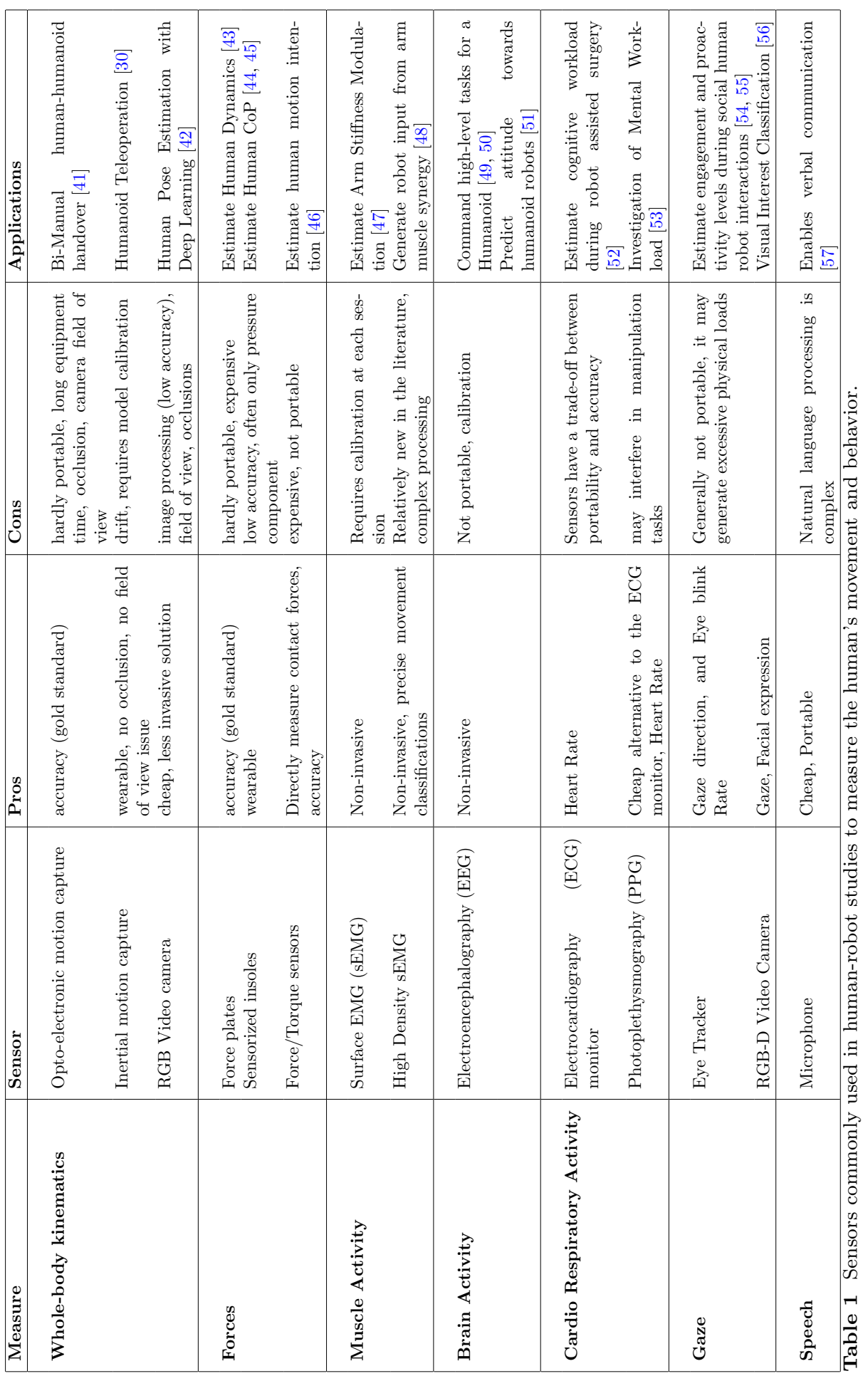


Measurements of physiological quantities are also common in human-robot cooperation. Physiological quantities can be used as such, for instance, electromyography (EMG) signals have been used to estimate human muscle fatigue [47]. But physiological quantities can also serve to estimate the human cognitive state: electrocardiography (ECG) and galvanic skin response (GSR) signals have been linked to stress and anxiety levels [58], while eye gazing was correlated with engagement and proactivity levels during social human robot interactions without physical contact [55].

In more recent work, there are indications that individual factors such as personality, can affect the human posture and motion while interacting with a humanoid robot [59] (motor contagion), or even the level of trust towards the robot [53]. These emotional, and perception factors should also be monitored by the robot in order to provide mental safety during interactions [60].

Finally, the above-mentioned measurements are often used in combination with a model of the human body, in order to retrieve further information. Many levels of details exist to represent the human body [61], but the most widely used in human-robot cooperation are the rigid body and musculoskeletal models. In rigid body models, the human body is represented as a kinematic chain of rigid segments linked together by ideal joints. This is typically done to estimate joint torques via inverse dynamics [43]. Such robotics-based models are also used to simulate human motion at a low computational cost [62]. Musculoskeletal models [63] include muscles and possibly tendons, providing a better degree of realism, obviously at a higher computational cost.

\section{Applications of humanoids interacting and cooperating with humans}

Humanoid robots are versatile platforms that can interact with and help humans in different contexts, relying on the cooperative and human-aware decision and control skills discussed in the previous sections. We discuss three main scenarios and provide examples of recent work listed in Table 2, reporting the type of robot and its control strategy used for each application.

\subsection{Humanoids as companions: coaches and education tools}

Humanoids endowed with social skills have the potential to assist humans in their daily endeavors (i.e., at a supermarket, at school, at work or at home) and as tools for education and rehabilitation. The anthropomorphic shape coupled with advanced cognitive and social behaviors, gestures and communication channels, can favor legibility, engagement, attunement and trust [51, 54].

The small humanoid NAO from Softbank Robotics is a representative example of a robot that has been employed in several researches as classmate [83] or tutor [84] in educational environments, as storyteller for children [85], 


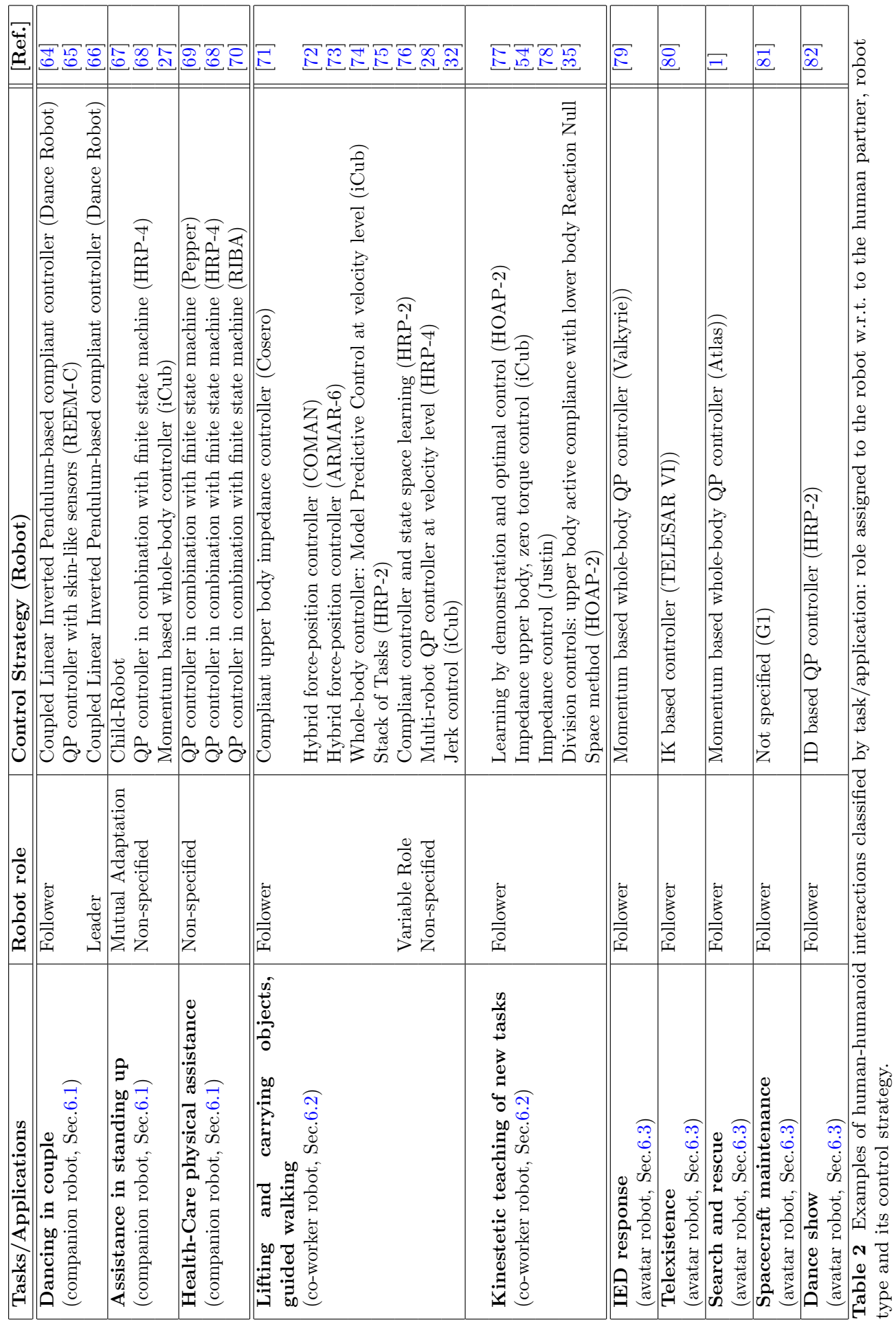


as fitness companion [86], as personal assistant in eating disorders[87] and diabetes management [88]. Such applications heavily rely on the socio-cognitive abilities of the humanoid that become an instrument for inducing a sustained engagement into a shared activity. Humanoid robots have been also successfully applied both as diagnosis instruments as well as remediation tools for neurodevelopmental deficits (e.g., autism and attention deficit/hyperactivity disorder), aging and neurodegenerative diseases such as Alzheimer [89].

Finally, humanoids could also be teleoperated to enable "distant" social interactions. Their human-like embodiment simplifies the projection of the human operator into the robotic body and can induce a sustained engagement into shared activities with the human partner in the remote site. More details about humanoid teleoperation are discussed later (Section 6.3).

\subsection{Humanoids as co-workers: optimizing the human ergonomics and performance}

Collaborative robots have received a lot of attention lately due to their potential to act as co-workers that can possibly improve working conditions. While initially focused on fixed-base robotic arms, research in this domain is now moving towards robotic manipulators mounted on wheeled mobile bases [90]. Even if providing more mobility, these robots remain largely limited to indoor settings with flat and uncluttered ground. Humanoid robots instead, could adapt to different environments and leverage their versatility. Even if their capabilities have been mostly validated in laboratory setups, the ultimate goal is to make them proactively work side by side humans without the need of protective cages [46]. To this end, they should also exhibit advanced interpersonal communication skills, and be able to learn new operations and new tasks through social interaction [91].

Even though most approaches proposed for robotic manipulators can be ported to humanoids with limited adaptation, examples of legged humanoids as co-workers in a physical interaction are still scarce. Most human-humanoid collaboration studies were focused on object carrying. For instance, Agravante et al. proposed a control framework that takes into consideration constraints for both walking and interacting with a human in a carrying task [46]. Otani et al. proposed to take into account the whole-body dynamics of the human to control an interacting humanoid robot [28]. Similarly to [92] with a mobile manipulator, Rapetti et al. proposes to improve human ergonomics by extending their humanoid whole-body controller to try to minimize the estimated torque, and joint velocities from a human partner [93].

To ease the balance issues faced in legged locomotion, humanoid torsos on wheels can also be used as co-workers [73]. Even though they do not possess legs, humans perceive them differently than robotic manipulators [59], which could have a beneficial impact depending on the application. In a recent work, Bolotnikova et al. presented a human-friendly humanoid that is able to approach a person in need and establish multimodal interactions for human assistance, including initiating physical contact [94]. Even though there was 
no shared task in [94] it gives a prime example of how leveraging the human perception could be used for assistance in the future.

\subsection{Humanoids as avatars: enabling humans to act in remote environments}

There are many scenarios where the human presence at the site is inherently dangerous (e.g. interventions in contaminated environments, construction sites, space exploration). In such situations, robots could be employed to replace humans at the site as physical avatars, protecting the operators from any potential hazards. Robot avatars could also be helpful in contexts not necessarily dangerous, for example to allow humans to virtually exist in another location in view of a more ecological and time-efficient society with an overall improved work-life balance.

A teleoperation system is a cooperative set-up where the robot imitates or replicates the human's actions to reach a common objective. First the human motion is captured and then converted in real-time into corresponding references for a whole-body controller that generates the joint or torque commands actuating the robot. The motion capture techniques are generally based on optical tracking or inertial technology (Section 5). Alternatively, the operator can directly control the robot through dual-arm exoskeletons [95] or wholebody exoskeleton cockpits [96], in which case their motion is tracked by the actuators and sensors of the device.

The captured human information is then transformed into corresponding values for the robot. A common retargeting method consists in performing an identity map between the rotational motion of the human and the robot, while using a fixed scaling factor for translational movements [97]. The joint angles and velocity of the human joints instead are either manually mapped to the corresponding joints of the robot [30], or automatically found by recurring to learning techniques [98].

The retargeted information is then corrected via feedback controllers [99] to ensure the dynamical balance of the teleoperated references on the humanoid robot. The resulting dynamically feasible references are then sent to the lowlevel controller, which is classically formulated as a QP [79], as explained in Section 4. The QP optimization is solved by taking into account the retargeted information as references, computing the actuating commands for the robot.

While controlling the robot, it is of crucial importance to sufficiently inform the operator of their avatar state, to give them the illusion of being physically present at the site, producing effective behaviors. A conventional way to provide situation awareness to the human operator is through visual feedback. The user can wear VR headsets, connected to the robot cameras or visualize on displays the information coming from the cameras of the robot together with images of the remote environment coming from LIDAR sensors and other external cameras. However, visual feedback is not sufficient for many real-world applications, especially those involving power manipulation or interaction with other human subjects. In such scenarios also the haptic feedback is required to 
exploit the human operator's motor skills in order to augment the robot performance. Force feedback, tactile and vibro-tactile feedback are the most used [100]. Voice and sound feedback can further enrich the virtual experience of the human operator especially in scenarios where other humans are present in the remote robot location [80]. Table 2 reports some examples of applications of teleoperated humanoid robots.

\section{Conclusion}

To guarantee proficient and adequate cooperative behaviors, humanoid robots need to advance their cognitive, social and physical interaction skills. This article reported on the current work in these areas of research, acknowledging the main limitations due to the real-time nature of the interaction and the complexity of modeling and identifying the human state. Human-aware humanoid collaborators capable of long-term interactions in real situations are the next grand challenge.

\section{Supplementary information. None.}

Acknowledgments. This work was supported by the European Union Horizon 2020 Research and Innovation Program under Grant Agreement No. 731540 (project AnDy), the European Research Council (ERC) under Grant Agreement No. 637972 (project ResiBots), the French Agency for Research under the ANR Grant No. ANR-18-CE33-0001 (project Flying Co-Worker) and the ANR-FNS Grant No. ANR-19-CE19-0029 - FNS 200021E_189475/1 (project iReCheck), the Inria-DGA grant ("humanoïde résilient"), and the Inria "ADT" wbCub/wbTorque.

\section{Compliance with Ethics Guidelines}

Conflict of Interest. The authors declare that they have no conflict of interest.

Human and Animal Rights and Informed Consent. This article does not contain any studies with human or animal subjects performed by any of the authors.

\section{References}

[1] Johnson, M., Shrewsbury, B., Bertrand, S., Wu, T., Duran, D., Floyd, M., Abeles, P., Stephen, D., Mertins, N., Lesman, A., Carff, J., Rifenburgh, W., Kaveti, P., Straatman, W., Smith, J., Griffioen, M., Layton, B., De Boer, T., Koolen, T., Pratt, J.: Team IHMC's lessons learned from the DARPA robotics challenge trials. Journal of Field Robotics 32 (2015). https://doi.org/10.1002/rob.21571 
[2] Kheddar, A., Caron, S., Gergondet, P., Comport, A., Tanguy, A., Ott, C., Henze, B., Mesesan, G., Englsberger, J., Roa, M.A., Wieber, P., Chaumette, F., Spindler, F., Oriolo, G., Lanari, L., Escande, A., Chappellet, K., Kanehiro, F., Rabat, P.: Humanoid robots in aircraft manufacturing: The airbus use cases. IEEE Robotics Automation Magazine 26(4), 30-45 (2019). https://doi.org/10.1109/MRA.2019.2943395

[3] Shigemi, S.: In: Goswami, A., Vadakkepat, P. (eds.) ASIMO and Humanoid Robot Research at Honda, pp. 1-36. Springer, Dordrecht (2018). https://doi.org/10.1007/978-94-007-7194-9_9-2

[4] Nelson, G., Saunders, A., Playter, R.: In: Goswami, A., Vadakkepat, P. (eds.) The PETMAN and Atlas Robots at Boston Dynamics, pp. 169-186. Springer, Dordrecht (2019). https://doi.org/10.1007/ 978-94-007-6046-2_15

[5] Digit, advanced mobility for the human world [online]. https://www.agilityrobotics.com/robots

[6] Lesort, T., Lomonaco, V., Stoian, A., Maltoni, D., Filliat, D., DazRodrguez, N.: Continual learning for robotics: Definition, framework, learning strategies, opportunities and challenges. Information Fusion 58, 52-68 (2020). https://doi.org/10.1016/j.inffus.2019.12.004

[7] Wood, R., Baxter, P., Belpaeme, T.: A review of long-term memory in natural and synthetic systems. Adaptive Behavior 20(2), 81-103 (2012). https://doi.org/10.1177/1059712311421219

[8] Saupp, A., Mutlu, B.: Robot deictics: How gesture and context shape referential communication. In: 2014 9th ACM/IEEE International Conference on Human-Robot Interaction (HRI), pp. 342-349 (2014). https://doi.org/10.1145/2559636.2559657

[9] Yogeeswaran, K., Złotowski, J., Livingstone, M., Bartneck, C., Sumioka, H., Ishiguro, H.: The interactive effects of robot anthropomorphism and robot ability on perceived threat and support for robotics research. J. Hum.-Robot Interact. 5(2), 29-47 (2016). https://doi.org/10.5898/ JHRI.5.2. Yogeeswaran

[10] Takayama, L., Dooley, D., Ju, W.: Expressing thought: Improving robot readability with animation principles. In: Proceedings of the 6th International Conference on Human-Robot Interaction. HRI '11, pp. 69-76. Association for Computing Machinery, New York, NY, USA (2011). https://doi.org/10.1145/1957656.1957674

[11] Vinciarelli, A., Pantic, M., Bourlard, H.: Social signal processing: Survey of an emerging domain. Image and Vision Computing 27(12), 1743-1759 
(2009). https://doi.org/10.1016/j.imavis.2008.11.007

[12] Breazeal, C.: Designing Sociable Robots. MIT Press, Cambridge, MA, USA (2002). https://doi.org/10.7551/mitpress/2376.001.0001. https://doi.org/10.7551/mitpress/2376.001.0001

[13] Scassellati, B.: Theory of mind for a humanoid robot. Autonomous Robots 12(1), 13-24 (2002). https://doi.org/10.1023/A:1013298507114

[14] Anzalone, S.M., Boucenna, S., Ivaldi, S., Chetouani, M.: Evaluating the engagement with social robots. International Journal of Social Robotics 7(4), 465-478 (2015). https://doi.org/10.1007/s12369-015-0298-7

[15] Thomas, F., Johnston, O., Thomas, F.: The Illusion of Life: Disney Animation. Hyperion New York, (1995)

[16] Bartneck, C., Kulić, D., Croft, E., Zoghbi, S.: Measurement instruments for the anthropomorphism, animacy, likeability, perceived intelligence, and perceived safety of robots. International Journal of Social Robotics 1(1), 71-81 (2009). https://doi.org/10.1007/s12369-008-0001-3

[17] Syrdal, D.S., Dautenhahn, K., Koay, K.L., Walters, M.L.: The negative attitudes towards robots scale and reactions to robot behaviour in a live human-robot interaction study. In: Adaptive and Emergent Behaviour and Complex Systems, pp. 109-115. SSAISB, (2009)

[18] Ramirez, M., Geffner, H.: Goal recognition over pomdps: Inferring the intention of a pomdp agent. In: IJCAI International Joint Conference on Artificial Intelligence, pp. 2009-2014 (2011)

[19] * Nikolaidis, S., Hsu, D., Srinivasa, S.: Human-robot mutual adaptation in collaborative tasks: Models and experiments. The International Journal of Robotics Research 36(5-7), 618-634 (2017).

This paper introduces a formalization for mutual adaptation between robot and a human in a collaborative task and shows how the proposed method can outperform precedent solutions in a human-robot team.

[20] Tabrez, A., Luebbers, M.B., Hayes, B.: A survey of mental modeling techniques in human-robot teaming. Current Robotics Reports, 1-9 (2020)

[21] Bestick, A., Bajcsy, R., Dragan, A.D.: Implicitly Assisting Humans to Choose Good Grasps in Robot to Human Handovers. In: 2016 International Symposium on Experimental Robotics vol. 1, pp. 341354. Springer, (2017). Series Title: Springer Proceedings in Advanced Robotics 
[22] Kaelbling, L.P., Littman, M.L., Cassandra, A.R.: Planning and acting in partially observable stochastic domains. Artificial Intelligence 101(1), 99-134 (1998)

[23] Silver, D., Veness, J.: Monte-carlo planning in large POMDPs. In: Lafferty, J.D., Williams, C.K.I., Shawe-Taylor, J., Zemel, R.S., Culotta, A. (eds.) Advances in Neural Information Processing Systems 23, pp. 2164-2172. Curran Associates, Inc., (2010)

[24] Nikolaidis, S., Hsu, D., Srinivasa, S.: Human-robot mutual adaptation in collaborative tasks: Models and experiments. The International Journal of Robotics Research 36(5-7), 618-634 (2017)

[25] Li, Y., Tee, K.P., Chan, W.L., Yan, R., Chua, Y., Limbu, D.K.: Continuous role adaptation for human-robot shared control. IEEE Transactions on Robotics 31(3), 672-681 (2015)

[26] Amodei, D., Olah, C., Steinhardt, J., Christiano, P.F., Schulman, J., Mané, D.: Concrete problems in ai safety. ArXiv abs/1606.06565 (2016)

[27] Romano, F., Nava, G., Azad, M., Čamernik, J., Dafarra, S., Dermy, O., Latella, C., Lazzaroni, M., Lober, R., Lorenzini, M., et al.: The codyco project achievements and beyond: Toward human aware wholebody controllers for physical human robot interaction. IEEE Robotics and Automation Letters 3(1), 516-523 (2017)

[28] * Otani, K., Bouyarmane, K., Ivaldi, S.: Generating assistive humanoid motions for co-manipulation tasks with a multi-robot quadratic program controller. In: 2018 IEEE International Conference on Robotics and Automation (ICRA), pp. 3107-3113 (2018).

This paper presents a multi-robot quadratic program controller which allows to keep the robot balanced, while also assisting the human in achieving their shared objectives.

[29] Dermy, O., Chaveroche, M., Colas, F., Charpillet, F., Ivaldi, S.: Prediction of human whole-body movements with ae-promps. In: 2018 IEEERAS 18th International Conference on Humanoid Robots (Humanoids), pp. 572-579 (2018)

[30] Penco, L., Scianca, N., Modugno, V., Lanari, L., Oriolo, G., Ivaldi, S.: A multimode teleoperation framework for humanoid loco-manipulation: An application for the icub robot. IEEE Robotics Automation Magazine 26(4), 73-82 (2019)

[31] Tirupachuri, Y., Nava, G., Rapetti, L., Latella, C., Pucci, D.: Trajectory advancement during human-robot collaboration. In: 2019 28th IEEE 
International Conference on Robot and Human Interactive Communication (RO-MAN), pp. 1-8 (2019)

[32] Gazar, A., Nava, G., Chavez, F..J.A., Pucci, D.: Jerk control of floating base systems with contact-stable parameterized force feedback. IEEE Transactions on Robotics (2020)

[33] Brygo, A., Sarakoglou, I., Tsagarakis, N., Caldwell, D.: Telemanipulation with a humanoid robot under autonomous joint impedance regulation and vibrotactile balancing feedback. (2014). https://doi.org/ 10.1109/HUMANOIDS.2014.7041465

[34] Ranatunga, I., Lewis, F.L., Popa, D.O., Tousif, S.M.: Adaptive admittance control for human-robot interaction using model reference design and adaptive inverse filtering. IEEE transactions on control systems technology 25(1), 278-285 (2016)

[35] Kormushev, P., Nenchev, D.N., Calinon, S., Caldwell, D.G.: Upper-body kinesthetic teaching of a free-standing humanoid robot. In: 2011 IEEE International Conference on Robotics and Automation, pp. 3970-3975 (2011)

[36] Bussy, A., Gergondet, P., Kheddar, A., Keith, F., Crosnier, A.: Proactive behavior of a humanoid robot in a haptic transportation task with a human partner. In: 2012 IEEE RO-MAN: The 21st IEEE International Symposium on Robot and Human Interactive Communication, pp. 962967 (2012)

[37] Mainprice, J., Sisbot, E.A., Jaillet, L., Cortés, J., Alami, R., Siméon, T.: Planning human-aware motions using a sampling-based costmap planner. In: 2011 IEEE International Conference on Robotics and Automation, pp. 5012-5017 (2011)

[38] Li, Y., Ge, S.S.: Human-robot collaboration based on motion intention estimation. IEEE/ASME Transactions on Mechatronics 19(3), 1007-1014 (2013)

[39] Jarrasse, N., Sanguineti, V., Burdet, E.: Slaves no longer: review on role assignment for human-robot joint motor action. Adaptive Behavior 22(1), 70-82 (2014)

[40] Buondonno, G., Patota, F., Wang, H., De Luca, A., Kosuge, K.: A model predictive control approach for the partner ballroom dance robot. In: 2015 IEEE International Conference on Robotics and Automation (ICRA), pp. 774-780 (2015) 
[41] Vasalya, A.: Human and Humanoid robot co-workers: motor contagions and whole-body handover. PhD thesis, Université de Montpellier (December 2019). https://hal.archives-ouvertes.fr/tel-02839897

[42] Zheng, C., Wu, W., Yang, T., Zhu, S., Chen, C., Liu, R., Shen, J., Kehtarnavaz, N., Shah, M.: Deep Learning-Based Human Pose Estimation: A Survey (2020)

[43] * Latella, C., Lorenzini, M., Lazzaroni, M., Romano, F., Traversaro, S., Akhras, M.A., Pucci, D., Nori, F.: Towards real-time whole-body human dynamics estimation through probabilistic sensor fusion algorithms. Autonomous Robots 43(6), 1591-1603 (2019).

The authors proposed a probabilistic framework and an estimation tool for online monitoring of the human dynamics during humanrobot collaboration tasks.

[44] Lorenzini, M., Kim, W., De Momi, E., Ajoudani, A.: A synergistic approach to the real-time estimation of the feet ground reaction forces and centers of pressure in humans with application to human-robot collaboration. IEEE Robotics and Automation Letters 3(4), 3654-3661 (2018)

[45] Sorrentino, I., Andrade Chavez, F.J., Latella, C., Fiorio, L., Traversaro, S., Rapetti, L., Tirupachuri, Y., Guedelha, N., Maggiali, M., Dussoni, S., et al.: A novel sensorised insole for sensing feet pressure distributions. Sensors 20(3), 747 (2020)

[46] * Agravante, D.J., Cherubini, A., Sherikov, A., Wieber, P.-B., Kheddar, A.: Human-humanoid collaborative carrying. IEEE Transactions on Robotics 35(4), 833-846 (2019).

This paper presents a framework for collaborative carrying based on whole-body controlling, the framework considers the taxonomy of the task, the roles of the agent, the walking pattern and the stabilization in presence of external forces.

[47] Peternel, L., Tsagarakis, N., Caldwell, D., Ajoudani, A.: Adaptation of robot physical behaviour to human fatigue in human-robot comanipulation. In: 2016 IEEE-RAS 16th International Conference on Humanoid Robots (Humanoids), pp. 489-494 (2016)

[48] Ison, M., Vujaklija, I., Whitsell, B., Farina, D., Artemiadis, P.: Simultaneous myoelectric control of a robot arm using muscle synergy-inspired inputs from high-density electrode grids. In: 2015 IEEE International Conference on Robotics and Automation (ICRA), pp. 6469-6474 (2015). https://doi.org/10.1109/ICRA.2015.7140108

[49] Li, W., Jaramillo, C., Li, Y.: Development of mind control system for 
humanoid robot through a brain computer interface. In: 2012 Second International Conference on Intelligent System Design and Engineering Application, pp. 679-682 (2012). https://doi.org/10.1109/ISdea.2012. 484

[50] Bell, C.J., Shenoy, P., Chalodhorn, R., Rao, R.P.: Control of a humanoid robot by a noninvasive brain-computer interface in humans. Journal of neural engineering 5(2), 214 (2008)

[51] Bossi, F., Willemse, C., Cavazza, J., Marchesi, S., Murino, V., Wykowska, A.: The human brain reveals resting state activity patterns that are predictive of biases in attitudes toward robots. Science Robotics 5(46) (2020) https://robotics.sciencemag.org/content/5/46/eabb6652.full.pdf. https://doi.org/10.1126/scirobotics.abb6652

[52] Zhou, T., Cha, J.S., Gonzalez, G., Wachs, J.P., Sundaram, C.P., Yu, D.: Multimodal physiological signals for workload prediction in robot-assisted surgery. ACM Transactions on Human-Robot Interaction (THRI) 9(2), 1-26 (2020)

[53] Hu, Y., Benallegue, M., Venture, G., Yoshida, E.: Interact with me: An exploratory study on interaction factors for active physical human-robot interaction. IEEE Robotics and Automation Letters 5(4), 6764-6771 (2020). https://doi.org/10.1109/LRA.2020.3017475

[54] Anzalone, S.M., Boucenna, S., Ivaldi, S., Chetouani, M.: Evaluating the engagement with social robots. International Journal of Social Robotics 7(4), 465-478 (2015)

[55] Baraglia, J., Cakmak, M., Nagai, Y., Rao, R., Asada, M.: Efficient human-robot collaboration: when should a robot take initiative? The International Journal of Robotics Research, 027836491668825 (2017). https://doi.org/10.1177/0278364916688253

[56] Risskov Sørensen, A., Palinko, O., Krüger, N.: Classification of visual interest based on gaze and facial features for human-robot interaction. In: Proceedings of the 16th International Joint Conference on Computer Vision, Imaging and Computer Graphics Theory and Applications. SCITEPRESS Digital Library, (2020)

[57] Cangelosi, A., Ogata, T.: Speech and Language in Humanoid Robots, pp. 1-32. Springer, Dordrecht (2016). https://doi.org/10.1007/ 978-94-007-7194-9_135-1

[58] Cruz-Maya, A., Agrigoroaie, R., Tapus, A.: Improving users performance 
by motivation: Matching robot interaction strategy with users regulatory state. In: International Conference on Social Robotics, pp. 464-473 (2017). Springer

[59] Vasalya, A., Ganesh, G., Kheddar, A.: More than just co-workers: Presence of humanoid robot co-worker influences human performance. PLOS ONE 13(11), 1-19 (2018). https://doi.org/10.1371/journal.pone. 0206698

[60] Kamide, H., Mae, Y., Kawabe, K., Shigemi, S., Hirose, M., Arai, T.: New measurement of psychological safety for humanoid. In: 2012 7th ACM/IEEE International Conference on Human-Robot Interaction (HRI), pp. 49-56 (2012). https://doi.org/10.1145/2157689.2157698

[61] Scataglini, S., Paul, G.: DHM and Posturography. Academic Press, (2019)

[62] Maurice, P., Padois, V., Measson, Y., Bidaud, P.: Human-oriented design of collaborative robots. International Journal of Industrial Ergonomics 57, 88-102 (2017)

[63] Peternel, L., Fang, C., Tsagarakis, N., Ajoudani, A.: A selective muscle fatigue management approach to ergonomic human-robot comanipulation. Robotics and Computer-Integrated Manufacturing 58, 69-79 (2019)

[64] Wang, H., Kosuge, K.: Control of a robot dancer for enhancing haptic human-robot interaction in waltz. IEEE transactions on haptics $5(3)$, 264-273 (2012)

[65] * Kobayashi, T., Dean-Leon, E., Guadarrama-Olvera, J.R., Bergner, F., Cheng, G.: Multi-contacts force-reactive walking control during physical human-humanoid interaction. In: 2019 IEEE-RAS 19th International Conference on Humanoid Robots (Humanoids), pp. 33-39 (2019).

This paper proposes a force-reactive walking control framework for stabilization during physical human-robot interaction where the contact forces are measured by robotic skin. The method has been tested on dancing task while teaching footsteps.

[66] Granados, D.F.P., Yamamoto, B.A., Kamide, H., Kinugawa, J., Kosuge, K.: Dance teaching by a robot: Combining cognitive and physical human-robot interaction for supporting the skill learning process. IEEE Robotics and Automation Letters 2(3), 1452-1459 (2017)

[67] Ikemoto, S., Amor, H.B., Minato, T., Ishiguro, H., Jung, B.: Physical interaction learning: Behavior adaptation in cooperative human-robot tasks involving physical contact. In: RO-MAN 2009-The 18th IEEE 
International Symposium on Robot and Human Interactive Communication, pp. 504-509 (2009)

[68] * López, A.M., Vaillant, J., Keith, F., Fraisse, P., Kheddar, A.: Compliant control of a humanoid robot helping a person stand up from a seated position. In: 2014 IEEE-RAS International Conference on Humanoid Robots, pp. 817-822 (2014).

This paper proposes a whole-body control framework to plan a stable initial posture for a humanoid robot supporting a person from sitting to standing while considering the patience degree of autonomy. Moreover the authors proposed a control law to make the robot keep a contact force and follow the motion of the person compliantly.

[69] * Bolotnikova, A., Courtois, S., Kheddar, A.: Autonomous initiation of human physical assistance by a humanoid. In: 2020 29th IEEE International Conference on Robot and Human Interactive Communication (RO-MAN), pp. 857-862.

Framework for physical assistance of a frail person based on whole body controller for autonomously reach a person, perform audiovisual communication of intent, and establish several physical contacts.

[70] Mukai, T., Hirano, S., Yoshida, M., Nakashima, H., Guo, S., Hayakawa, Y.: Tactile-based motion adjustment for the nursing-care assistant robot riba. In: 2011 IEEE International Conference on Robotics and Automation, pp. 5435-5441 (2011)

[71] Stückler, J., Behnke, S.: Following human guidance to cooperatively carry a large object. In: 2011 11th IEEE-RAS International Conference on Humanoid Robots, pp. 218-223 (2011)

[72] Lanini, J., Razavi, H., Urain, J., Ijspeert, A.: Human intention detection as a multiclass classification problem: Application in physical humanrobot interaction while walking. IEEE Robotics and Automation Letters 3(4), 4171-4178 (2018)

[73] Asfour, T., Waechter, M., Kaul, L., Rader, S., Weiner, P., Ottenhaus, S., Grimm, R., Zhou, Y., Grotz, M., Paus, F.: Armar-6: A high-performance humanoid for human-robot collaboration in real-world scenarios. IEEE Robotics \& Automation Magazine 26(4), 108-121 (2019)

[74] Bombile, M., Billard, A.: Capture-point based balance and reactive omnidirectional walking controller. In: 2017 IEEE-RAS 17th International Conference on Humanoid Robotics (Humanoids), pp. 17-24 (2017)

[75] Stasse, O., Evrard, P., Perrin, N., Mansard, N., Kheddar, A.: Fast 
foot prints re-planning and motion generation during walking in physical human-humanoid interaction. In: 2009 9th IEEE-RAS International Conference on Humanoid Robots, pp. 284-289 (2009)

[76] Evrard, P., Gribovskaya, E., Calinon, S., Billard, A., Kheddar, A.: Teaching physical collaborative tasks: object-lifting case study with a humanoid. In: 2009 9th IEEE-RAS International Conference on Humanoid Robots, pp. 399-404 (2009)

[77] Calinon, S., Guenter, F., Billard, A.: On learning, representing, and generalizing a task in a humanoid robot. IEEE Transactions on Systems, Man, and Cybernetics, Part B (Cybernetics) 37(2), 286-298 (2007)

[78] Lee, D., Ott, C., Nakamura, Y., Hirzinger, G.: Physical human robot interaction in imitation learning. In: 2011 IEEE International Conference on Robotics and Automation, pp. 3439-3440 (2011)

[79] Jorgensen, S.J., Lanighan, M.W., Bertrand, S.S., Watson, A., Altemus, J.S., Askew, R.S., Bridgwater, L., Domingue, B., Kendrick, C., Lee, J., et al.: Deploying the nasa valkyrie humanoid for ied response: An initial approach and evaluation summary. 2019 IEEE-RAS 19th International Conference on Humanoid Robots (Humanoids) (2019). https://doi.org/ 10.1109/humanoids43949.2019.9034993

[80] Tachi, S.: Telexistence. World Scientific 2nd edition, (2015)

[81] GITAI Partners With JAXA to Send Telepresence Robots to Space [online]. https://spectrum.ieee.org/automaton/robotics/spacerobots/gitai-partners-with-jaxa-to-send-telepresence-robots-to-space

[82] Ramos, O.E., Mansard, N., Stasse, O., Benazeth, C., Hak, S., Saab, L.: Dancing humanoid robots: Systematic use of osid to compute dynamically consistent movements following a motion capture pattern. IEEE Robotics Automation Magazine 22(4), 16-26 (2015). https://doi.org/10. 1109/MRA.2015.2415048

[83] Hamamsy, L.E., Johal, W., Asselborn, T., Nasir, J., Dillenbourg, P.: Learning by collaborative teaching: An engaging multiparty cowriter activity. In: 2019 28th IEEE International Conference on Robot and Human Interactive Communication (RO-MAN), pp. 1-8 (2019). https://doi.org/10.1109/RO-MAN46459.2019.8956358. https://doi.org/10.1109/RO-MAN46459.2019.8956358

[84] Chang, C.-W., Lee, J.-H., Chao, P.-Y., Wang, C.-Y., Chen, G.-D.: Exploring the possibility of using humanoid robots as instructional tools for teaching a second language in primary school. Journal of Educational Technology \& Society 13(2), 13-24 (2010) 
[85] Wong, C.J., Tay, Y.L., Wang, R., Wu, Y.: Human-robot partnership: A study on collaborative storytelling. In: 2016 11th ACM/IEEE International Conference on Human-Robot Interaction (HRI), pp. 535-536 (2016). https://doi.org/10.1109/HRI.2016.7451843. https://doi.org/10.1109/HRI.2016.7451843

[86] Görer, B., Salah, A.A., Akın, H.L.: A robotic fitness coach for the elderly. In: Augusto, J.C., Wichert, R., Collier, R., Keyson, D., Salah, A.A., Tan, A.-H. (eds.) Ambient Intelligence, pp. 124-139. Springer, Cham (2013). https://doi.org/10.1007/978-3-319-03647-2_9

[87] Robinson, N.L., Connolly, J., Hides, L., Kavanagh, D.J.: Social robots as treatment agents: Pilot randomized controlled trial to deliver a behavior change intervention. Internet Interventions 21, 100320 (2020). https:// doi.org/10.1016/j.invent.2020.100320

[88] Lau, Y., Chee, D.G.H., Chow, X.P., Wong, S.H., Cheng, L.J., Lau, S.T.: Humanoid robot-assisted interventions among children with diabetes: A systematic scoping review. International Journal of Nursing Studies 111, 103749 (2020). https://doi.org/10.1016/j.ijnurstu.2020.103749

[89] Pennisi, P., Tonacci, A., Tartarisco, G., Billeci, L., Ruta, L., Gangemi, S., Pioggia, G.: Autism and social robotics: A systematic review. Autism Research 9(2), 165-183 (2016). https://doi.org/10.1002/aur.1527

[90] Kim, W., Balatti, P., Lamon, E., Ajoudani, A.: Moca-man: A mobile and reconfigurable collaborative robot assistant for conjoined humanrobot actions. In: 2020 IEEE International Conference on Robotics and Automation (ICRA), pp. 10191-10197 (2020)

[91] Yokoyama, K., Handa, H., Isozumi, T., Fukase, Y., Kaneko, K., Kanehiro, F., Kawai, Y., Tomita, F., Hirukawa, H.: Cooperative works by a human and a humanoid robot. In: 2003 IEEE International Conference on Robotics and Automation (Cat. No. 03CH37422), vol. 3, pp. 2985-2991 (2003)

[92] Kim, W., Lorenzini, M., Balatti, P., Wu, Y., Ajoudani, A.: Towards ergonomic control of collaborative effort in multi-human mobile-robot teams. In: 2019 IEEE/RSJ International Conference on Intelligent Robots and Systems (IROS), pp. 3005-3011 (2019). https://doi.org/10. 1109/IROS40897.2019.8967628

[93] Tirupachuri, Y., Nava, G., Ferigo, D., Tagliapietra, L., Latella, C., Nori, F., Pucci, D.: Towards partner-aware humanoid robot control under physical interactions. In: IntelliSys (2019)

[94] Bolotnikova, A., Courtois, S., Kheddar, A.: Autonomous initiation 
of human physical assistance by a humanoid. In: 2020 29th IEEE International Conference on Robot and Human Interactive Communication (RO-MAN), pp. 857-862 (2020). https://doi.org/10.1109/ RO-MAN47096.2020.9223519

[95] Abi-Farrajl, F., Henze, B., Werner, A., Panzirsch, M., Ott, C., Roa, M.A.: Humanoid teleoperation using task-relevant haptic feedback. In: 2018 IEEE/RSJ International Conference on Intelligent Robots and Systems (IROS), pp. 5010-5017 (2018)

[96] Ishiguro, Y., Makabe, T., Nagamatsu, Y., Kojio, Y., Kojima, K., Sugai, F., Kakiuchi, Y., Okada, K., Inaba, M.: Bilateral humanoid teleoperation system using whole-body exoskeleton cockpit TABLIS. IEEE Robotics and Automation Letters 5(4), 6419-6426 (2020)

[97] * Y. Ishiguro, Kojima, K., Sugai, F., Nozawa, S., Kakiuchi, Y., Okada, K., Inaba, M.: High speed whole body dynamic motion experiment with real time master-slave humanoid robot system. In: 2018 IEEE International Conference on Robotics and Automation (ICRA), pp. 1-7 (2018). https://doi.org/10.1109/ICRA.2018.8461207.

This paper proposes a whole body master-slave control technique for online teleoperation of a life-sized humanoid robot.

[98] Villegas, R., Yang, J., Ceylan, D., Lee, H.: Neural kinematic networks for unsupervised motion retargetting. In: Proceedings of the IEEE Conference on Computer Vision and Pattern Recognition, pp. 8639-8648 (2018)

[99] Englsberger, J., Werner, A., Ott, C., Henze, B., Roa, M.A., Garofalo, G., Burger, R., Beyer, A., Eiberger, O., Schmid, K., et al.: Overview of the torque-controlled humanoid robot toro. In: 2014 IEEE-RAS International Conference on Humanoid Robots, pp. 916-923 (2014)

[100] Brygo, A., Sarakoglou, I., Garcia-Hernandez, N., Tsagarakis, N.: Humanoid robot teleoperation with vibrotactile based balancing feedback. In: Auvray, M., Duriez, C. (eds.) Haptics: Neuroscience, Devices, Modeling, and Applications, pp. 266-275. Springer, Berlin, Heidelberg (2014) 